\title{
Analysis of Stationary Means of Measurement Filters with Optimum Sensitivity
}

\author{
Andrei V. Kochetkov \\ Perm National Research Polytechnic University \\ Perm, Russia
}

Oleg V. Zakharov

Yuri Gagarin State Technical University of Saratov Saratov, Russia

\author{
Nikolaj M. Bobrovskij \\ Togliatti State University \\ Togliatti, Russia
}

\author{
Pavel A. Melnikov \\ Togliatti State University \\ Togliatti, Russia \\ Igor N. Bobrovskij \\ Togliatti State University \\ Togliatti, Russia
}

\author{
Vadim A. Kushnikov \\ Institute of Precision Mechanics and Control of RAS \\ The Russian Academy of Sciences \\ Saratov, Russia
}

\begin{abstract}
When transmitting information and control signals to electrical, electromechanical and mechatronic systems are require reduction to the lowest possible levels of noise interference. For this is substantiated in the general formulation of the one-dimensional problem of determining the sensitivity of a linear stationary optimal Wiener filtration. It is proposed to use the method of local approximation of the spectral densities for the quantitative determination of indicators of sensitivity converting electrical signals from the means of measurement. In relation to the different models produced recommendations on the advisability of accounting the characteristics of the signal deviations from nominal, deviations from the optimal structures and parameters for their implementation and configuration of the filters themselves.
\end{abstract}

Keywords - electrical signal; stationary filters; sensitivity; frequency characteristics; measuring instruments; optimization

\section{INTRODUCTION}

In many applied problems, for example, the transmission of information and control signals to electrical, electromechanical and mechatronic systems, which are presented in the form of open or closed structures, required reduction to the lowest possible levels of noise interference. Natural smoothing of noise in amplifier circuits and signal conversion devices often is not enough. It is therefore necessary to use smoothing or narrow-band filters with specially selected frequency characteristics.

In various fields of science to the quantitative indicators of influence on the qualitative and quantitative factors, used different basic concepts: coefficients and functions influence (metrology [1]), the sensitivity coefficients (in automatic control theory $[2,3]$ ), elasticity coefficients (in the economy). However, there are serious inconsistencies. In particular, in metrology «sensitivity» refers to the measurement of a constant value of a physical quantity, and interpreted, for example, as a «means of measurement property, defined by the ratio of the change in the means of the output signal to the calling of his change of the measured value». The sensitivity of the automatic control theory applies only to dynamic properties and characteristics (in particular, to the margin of stability, vibrational, etc.). With regard to the topic of this work is important one aspect, when evaluated noise immunity. At the time, it was necessary to create a common sensitivity theory in Russian foundations of the theory presented in [4].

With regard to the sensitivity of optimality imposes its own specificity [4]. Most of the publications, related to the sensitivity of the optimal economic solutions, are obtained by linear or non-linear programming. In these cases, the optimal solution is obtained with limited but finite deviations of the source data. The smallness of the impact of changes in indicators, insensitivity in relation to the managed systems with limited parameter changes qualifies as the attribute robustness of the system [6]. But this is not consistent with the interpretation of the term "robustness" in the fundamental work, for example, [7]. In [8] concept of robustness is given a specific meaning which is characterized in that the device is based on a problems description language for the logarithmic frequency characteristics. Quantitative accounting of the impact of various factors on the qualitative and quantitative indicators in different applications carried in parallel and almost independently in various fields. Almost always in the major integrated research took into quantitative account the uncertainty of conditions and input data for the objectives and parameters.

\section{Formulation OF THE PROBLEM}

Field researches of sensitivity properties in this paper is characterized by the following factors and obtained for them quantitative estimates: 
a) the object for which is determined by the sensitivity (you need to determine what sensitivity), changes in estimated optimal frequency characteristic (structure, parameter) or dispersion (or both, in different forms: absolute and relative percentage);

b) with respect to changes of any factor or factors and what is indicators factors, which vary by objective circumstances, evaluated the sensitivity and the method of setting changes (parametric or limitations areas); mostly it is the spectral density of the useful signal and noise;

c) under what conditions (if there is a relationship between changes in the spectral densities, whether used in the filter of the changes the spectral densities of information); necessary to determine whether for changes the frequency characteristic of filter are carried out according to the modified spectral densities or not.

The general formulation is considered a one-dimensional problem of determining the sensitivity of a linear stationary filtration optimal Wiener [9] using method of local approximation of spectral densities. In particular we used straightening asymptotic curves of the spectral density in logarithmic grid. The original formulation of the optimal stationary problem is presented the diagram in Fig. 1.

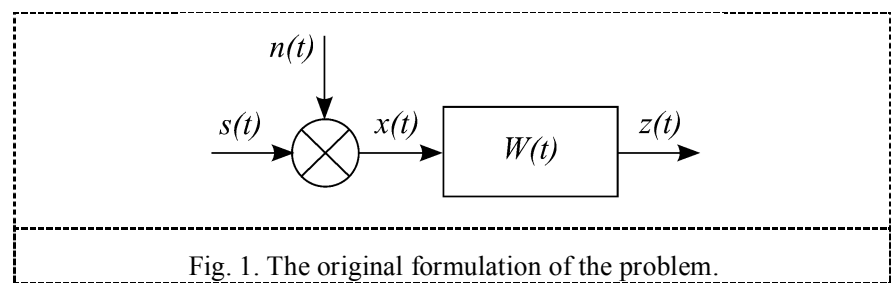

Below are the details of the known solution, [10] optimization problem with use of local approximations of spectral density of the useful signal and the noise. Input of the filter with the transfer function $W(j \omega)$ receives the signal $x(t)$, representing the additive sum of the useful signal $s(t)$ and noise $n(t)$ :

$$
x(t)=s(t)+n(t)
$$

The useful signal and the noise are considered to be nondeterministic random and their properties are given spectral densities $S_{s}(\omega)$ and $S_{n}(\omega)$. It is assumed that the dimension of noise given to the dimension of the desired signal. It is required that the filter output signal $z(t)$ may more accurately reproduce the desired signal $s(t)$. Accuracy of transformation is characterized by an error:

$$
e(t)=z(t)-s(t)
$$

Currently available CMM solutions help measure parts fast and with high precision, and can identify departures from preset shapes, keep measuring data statistics, and calculate tuning parameters needed to adjust the processing process using computers. On the other hand, a whole range of issues related to control of geometrical characteristics of complex surfaces still await resolution. Absence of an integrated mathematical foundation remains the greatest problem, therefore multiple separate mathematical models are necessary for the control process.
With regard to the spectral theory of random processes is usually administered assumption of stationary of both components as a function of time. However, we have found that the properties of the spectral densities of view it is quite possible, if they are not stationary, but can be represented as a result of passing through the stationary processes, stationary filters (e.g., is the integral of stationary processes). The criterion of accuracy is the standard deviation $\sigma_{\mathrm{e}}$. Along with this, is interested the ratio of standard deviations $\sigma_{\mathrm{e}} / \sigma_{s}$ (usually a percentage). Properties of the desired optimal transformation with a filter set frequency characteristic $W(j \omega)$, is chosen from the condition minimum of established variance $\sigma_{\mathrm{e}}^{2}$ :

$$
\sigma_{e}^{2}=(2 \pi)^{-1} \int_{-\infty}^{+\infty}\left\{S_{S}(\omega)(1-W(j \omega))^{2}+S_{n}(\omega) W(j \omega)^{2}\right\} d \omega \rightarrow \min
$$

In practice there are problems when the subject is not the definition of the useful signal, and the results of its transformation (e.g., derivative, i.e. the rate of change, or the integral of it). Such problems are common in navigation (passing from acceleration to velocity and displacement and vice versa). In this case, instead of (3) are written criteria for minimum squares of other quantities. Unfortunately, optimal definition of the useful signal at the same time does not mean optimal definition of the conversion results (derivatives or integrals), and synthesis of problems need to formulate and solve independently. Often, the procedure is similar to the solution, but the results are differing. It may be that for such requirements modified an established variance of the error does not exist. For example, if is to be determined the integral on the stationary of the useful signal, in the presence of noise with the usual properties of the error variance will always increase. In determining the derivative for conventional models often does not get the final dispersion of errors due to non-decreasing spectral density at $\omega \rightarrow \infty$. To obtain meaningful results in such cases it is necessary to change the signal model.

\section{MATHEMATICAL ModeL}

It is known that up to a factor variance of the error is represented as area under the envelope of the bottom curves of the spectral density of the useful signal and noise, if the curves are constructed in real. The solution $W(j \omega)$ for optimum filter is represented in the form of a rational function whose poles (roots of the denominator) are in the upper plane of the complex plane $\omega$. To determine the numerator can be used different procedures, it is often sufficient to impose the natural condition of the limb error variance. By formulation the same solution is reduced to stabilize the output of the parameter $z(t)$ of the object $\mathrm{O}$ in a closed system with negative feedback (Fig. 2).

Where $G(j \omega)$ - frequency response of the object $\mathrm{O}, K(j \omega)$ to be determined the frequency response of the controller $\mathrm{P}$, the output signal $u(t)$ which is a control action on the object, the state variable is $z(t)$ to be stabilizing around a constant value. It is assumed that the state parameter is directly measured, $n(t)$ - a measurement error, which also has the properties of noise. There is a perturbation effects $y(t)$ at the input of the object, assumed to be known spectral density 
$S_{y}(\omega)$ and $S_{n}(\omega)$. The idea of stabilization by feedback is reduced to the fact that the control action should maximize compensate adverse effects so as to reduce the output parameter of an object (more precisely to stabilize with respect to zero). Therefore, the criterion is used:

$$
\sigma_{z}^{2}=\min \text {. }
$$

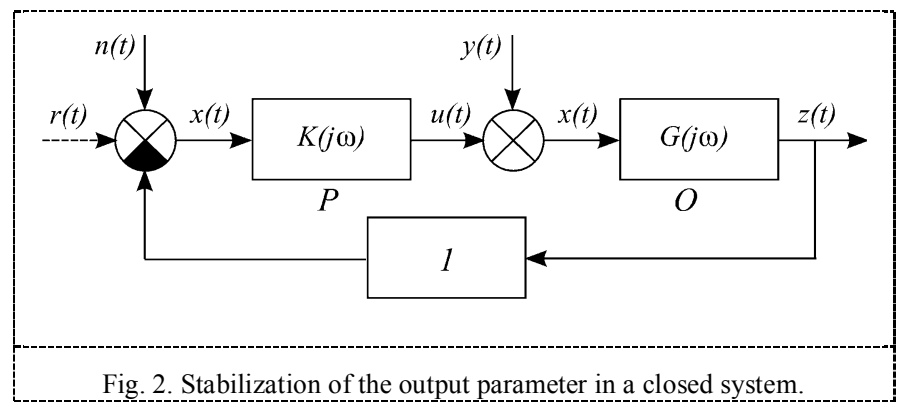

Equivalent structural transformation of the circuit in Fig. 2 is a scheme of Fig. 1 in which all impacts are applied to the closed system as a single whole. Thus, the circuit of scheme Fig. 2 shows transformation of the desired signal and noise amount, and plays the role of the useful signal converted to the output of perturbation effects object $G(t)$, and the role of noise $n(t)$. The optimization is performed for the entire closed system, based on the minimum requirements of dispersion output parameter. After finding the frequency characteristics in $W(j \omega)$ from the relationship

$$
K(j \omega)=W(j \omega)\{G(j \omega)[1-W(j \omega)]\}^{-1}
$$

is determined by the desired frequency response of the controller $K(j \omega)$. In the future, we consider the problem of synthesis of optimal transformation for the initial filtering circuit in Fig. 1. The results of calculations for typical model problems demonstrate that the resulting frequency response of the regulator to $K(j \omega)$ in closed systems in the form and structure have nothing to do with the optimal frequency characteristic [10]. Likewise, the conversion is carried out circuit with negative feedback of a more general form (Fig. 3), which has many elements.

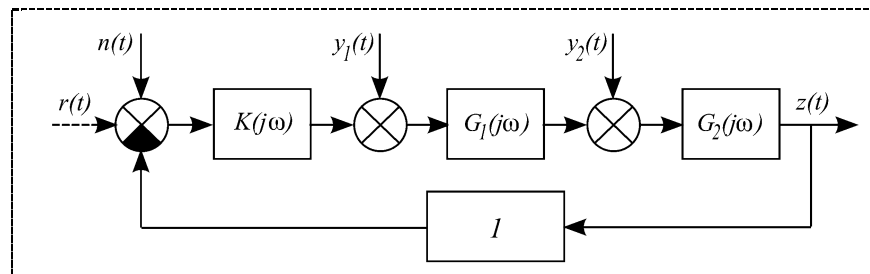

Fig. 3. The circuit with negative feedback.

In carrying out this scheme to open filter Fig. 3 exposure to the regulator, the frequency response is $K(j \omega)$ to be determined to play a role noise, and after the control - play the role of a useful signal components. After bringing the summation is carried out according to the rules set forth in [10], namely, the spectral density is defined as the envelope on top of all the components.
Common approaches to the analysis of the sensitivity to filtering problems are invariant with respect to methods for solving optimization problems. However, this paper examines the issues of sensitivity in relation to the fixed filters, the optimal steady state, as using local approximations decisions are often represented in the form of foreseeable and easy to analyze. When optimizing the filter and the synthesis of Fig. 1 of the desired pole frequency characteristic $W(j \omega)$ are the roots of the equation:

$$
S_{s}(\omega)+S_{n}(\omega)=0
$$

When the spectral density are not peaks and valleys and a logarithmic grid according to the $S_{s}(\omega)$ and $S_{n}(\omega)$ is approximately represented by broken lines with slopes of plots with coefficients that are multiples of $40 \mathrm{~dB} /$ decade. In the vicinity of the intersection point is convenient to use local, linear in the logarithmic power-grid approximation of the spectral densities:

$$
S_{s}(\omega) \approx A^{2} \omega^{-2 k} ; S_{n}(\omega) \approx B^{2} \omega^{2 l},
$$

where $k>0$ (useful signals are generally low frequency or slowly varying) for typical applications. The coefficient 1 , which can be either positive or negative, is often assumed to be zero (white noise model). This corresponds to an analytical representation of straightening curves of the spectral density near the point of intersection, and the method is called straightened frequency characteristics and spectral densities.

Intersection points may be several, but the typical case is when one point and the intersection of the desired signal spectral density curve going from top to bottom. In total, there are $2(k+l)$ roots of equation $(6)$ or the equation in this case:

$$
A^{2} \omega^{-2 k}+B^{2} \omega^{2 l}=0
$$

which are located on the complex plane uniformly on a circle of radius $\rho=(A / B)^{k+l}$. Those $\omega=\omega_{1}, \omega_{2}, \ldots, \omega_{k+l}$, which lie in the upper half of the complex plane of $\omega$, are poles (roots of the denominator) of the desired frequency characteristic of the optimal filter. The polynomials of the form

$$
P_{n}(\omega)=\left(\omega-\omega_{1}\right) \cdot\left(\omega-\omega_{2}\right) \ldots\left(\omega-\omega_{n}\right)
$$

called Butterworth polynomials, and filters, frequency characteristics of which are the denominators of this type Butterworth filters. Thus, the procedure for determining the poles (roots of the denominator) of the desired optimal frequency characteristic is quite simple and unified. Numerator of frequency characteristic often uniquely is determined by the conditions of finiteness of the variance error.

Of interest are the situations in which the curves of spectral densities have sharp peaks or dips. Traditional problems are transmitting narrowband signals and navigation signal theory (taking into account the curvature of the Earth, when it is possible to formulate a condition Schuler towards accelerated movement). Situations in which at the same spectral density of the useful signal and the noise and the same frequency, it is necessary to evaluate the useful signal itself, or, or its derivative, or its integral. Similar problems are encountered, for example, when evaluating navigation linear velocity, acceleration or displacement. In these cases, the frequency characteristics of the denominators are the same, and the numerators - different. 
There may be situations that determine the behavior of the spectral density of the useful signal and noise, as well as view transactions, linking the useful signal to the desired output (eg, differentiation or integration), in which no conversion makes it possible to avoid the increase of error variance, and set the variance does not exist. On the other hand, when the steady variance required conversion error exists, there is insufficient use of local approximations of spectral densities. If interference is a white noise, the problem of determining a useful signal with the spectral density $S_{s}(\omega) \approx A^{2} \omega^{-2 k}$ has a unique solution. The optimum filter is a Butterworth polynomial in the denominator of degree $k$, and the numerator is uniquely determined from the condition of finiteness of the variance error. For example, for $k=3$, the frequency characteristic has the form:

$W(j \omega)=\left[2 \rho(j \omega)^{2}+2 \rho^{2}(j \omega)+\rho^{3}\right]\left[(j \omega)^{3}+2 \rho(j \omega)^{2}+2 \rho^{2}(j \omega)+\rho^{3}\right]^{-1} .(10)$

In applied problems there is always a natural uncertainty of initial data. The problems of transformation of signals, when from the very beginning has open system, the uncertainty refers only to the signal properties. The problems of stabilization of output parameters of uncertainty relate to the structure and parameters of the system (i.e., transfer functions). After driving circuit with feedback to the base open loop Fig. 1 examines the impact of changes in the frequency response coefficients $W(j \omega)$, but then they need to be associated with possible changes in the parameters or control, or an object, or both. Sometimes it is advisable to carry out the reverse structural transformation from an open to a closed circuit c negative feedback, as this scheme can easily be read. Practical advantages of constructing a feedback scheme lie in the fact that the required high accuracy static (constant value during playback) is sufficient to have only a high stability in the feedback element. However, such a reduction to a closed circuit is ambiguous. Conveniently schemes lead to a form that the feedback was single, and in a straight chain were integrators.

\section{CONCLUSION}

The decision of problems of the optimal steady state of dynamic transformation the electrical signal measurement means of electrical, electromechanical and mechatronic devices in practice should be accompanied by a versatile and multifaceted study of the impact of deviations from the nominal frequency as the properties (spectral density) of the desired signal and noise, and the filter transfer function. Carried out researches allow in relation to the different models of these deviations are obtained with specific recommendations on the desirability or necessity of taking them into account in the implementation and configuration of filters.

\section{Acknowledgment}

This work was supported by Russian Foundation for Basic Research (project No. 15-08-08411 a).

\section{References}

[1] ISO/IEC Guide 99:2007. International vocabulary of metrology - Basic and general concepts and associated terms.

[2] E.N. Rosenwasser and R.M. Yusupov. The sensitivity of the automatic control systems. Leningrad. Energy, 1969. (on russian language).

[3] B.T. Polyak and P.S. Shcherbakov. Robust stability and control. Moscow. Science, 2002. (on russian language).

[4] R. Tomovic, Vukobratovic M. Overall sensitivity theory. Moscow. Peace. 1972. (on russian language).

[5] A.F. Izmailov. Sensitivity optimization. Moscow. Fizmatlit. 2006. (on russian language).

[6] V.O. Nikiforov. Adaptive and robust control with feedforward compensation. St. Petersburg. The science. 2003. (on russian language).

[7] Peter J. Huber. Robust Statistics. Wiley, 1981 (republished in paperback, 2004).

[8] V.A. Besekersky and A.V. Nebylov. Robust automatic control system. Moscow. Science, 1983. (on russian language).

[9] O.A. Stepanov. Fundamentals of estimation theory with applications to problems of navigation information processing. St. Petersburg. Electrical Appliance. 2012. (on russian language).

[10] I.B. Chelpanov. Optimal processing of signals in navigation systems. Moscow. Science. 1966. (on russian language).

[11] B.S. Brazhkin and V.S. Mirotvorskii. Calculation of Curved Surfaces on Coordinate Measuring Machines. Measurement Techniques. 2003, Volume 48, Issue 7, pp. 657-662.

[12] Lemeshko B. Yu. Errors when using nonparametric fitting criteria. Measurement Techniques. 2004, Volume 47, pp. 134-142.

[13] Frank R. Hampel, Elvezio M. Ronchetti, Peter J. Rousseeuw, Werner A. Stahel. Robust Statistics: The Approach Based on Influence Functions. 1986. John Wiley. 